\title{
Competitive Coexistence in a Two-Strain Epidemic Model with a Periodic Infection Rate
}

\author{
Chentong Li, Yingying Zhang, and Yicang Zhou \\ School of Mathematics and Statistics, Xi'an Jiaotong University, Xi'an 710049, China \\ Correspondence should be addressed to Yicang Zhou; zhouyc@xjtu.edu.cn
}

Received 22 September 2019; Accepted 6 February 2020; Published 10 March 2020

Academic Editor: Yukihiko Nakata

Copyright (C) 2020 Chentong Li et al. This is an open access article distributed under the Creative Commons Attribution License, which permits unrestricted use, distribution, and reproduction in any medium, provided the original work is properly cited.

In this article, we study the global dynamical behavior of a two-strain SIS model with a periodic infection rate. The positivity and boundedness of solutions are established, and the competitive exclusion conditions are given for the model. The conditions for the global stability of the disease-free equilibrium and persistence of the model are obtained. The conditions of coexistence in this model are also found. Finally, the conditions of uniqueness of the solution are proved.

\section{Introduction}

Since the first work [1] about the mathematical epidemic model published, there were plenty of results of infectious diseases in modeling and dynamics. These epidemic models usually include two important parameters, the infection rate and recovery rate. Many of the epidemic models focus on the disease eradication and persistence. Among these models, some are applied successfully on infectious disease forecast and control [2-6]. In the analysis of the epidemic model, it is found that infectious diseases often fluctuate over time and exhibit periodic behavior [7-9]. This fluctuation is also observed in the real data $[10,11]$. The influenza data from CDC [10] show that the number of influenza cases per week oscillates with a period between two peaks of one year and measles data [11] illustrate the period of two years. The periodic behavior of the incidence of many infectious diseases is caused by the influence of temperature and humidity on virus $[12,13]$. In $[14,15]$, the authors introduced the epidemic models that had periodic coefficients to illustrate the periodical phenomenon. An epidemic SIS model with a periodic infection rate was first considered by Hethcote [15]. Furthermore, Dietz [14] considered SIR and SEIR models with a periodic infection rate.
The causative agents of many diseases are represented by multiple genetically distinct variants. Early autonomous multistrain models suggested that competitive exclusion is the only possible result of the competition of many strains [16]. However, these models disregard many mechanisms such as coinfection [17], mutation [18], cross immunity [19], and periodicity [20], which were proposed as possible mechanisms that could support diversity of causative agents. Castillo-Chavez et al. [21] established a sexually transmitted disease model with two competing strains and found the conditions of coexistence equilibrium and its global stability. The interesting work finished by Nuño et al. [19] considered a two-strain influenza model with cross immunity, and their simulation results showed that there might be up to four coexistence equilibria for their model. Another result about multiplestrain model was finished by Martcheva [20]. In this work, the author introduced a nonautonomous multistrain epidemic model without susceptible individuals and found the coexistence and persistence conditions of this model. All of these results also show that the analysis of multiple-strain model can extend our knowledge about the mechanism of the multiple-strain coexistence and competitive exclusion. 
In this paper, we investigate a two-strain SIS model with a periodic infection rate

$$
\left\{\begin{array}{l}
\frac{\mathrm{d} S}{\mathrm{~d} t}=\lambda-\frac{\beta_{1}(t) S I_{1}}{1+S}-\frac{\beta_{2}(t) S I_{2}}{1+S}+\gamma_{1} I_{1}+\gamma_{2} I_{2}-\mathrm{d} S \\
\frac{\mathrm{d} I_{1}}{\mathrm{~d} t}=\left(\frac{\beta_{1}(t) S}{1+S}-k_{1}\right) I_{1}, \\
\frac{\mathrm{d} I_{2}}{\mathrm{~d} t}=\left(\frac{\beta_{2}(t) S}{1+S}-k_{2}\right) I_{2},
\end{array}\right.
$$

where $S$ is the number of susceptible individuals and $I_{i}(i=$ $1,2)$ is the number of individuals that are infected by the disease strain i. $0<\beta_{i}(t)<M(i=1,2)$ is the infection rate of strain $i$, and it is a periodic function with the period $T$. " $M$ " here is the upper bound of $\beta_{i}(t)(i=1,2) . d>0$ is the death rate of susceptible individuals. $k_{i}=\gamma_{i}+d+\alpha_{i}>0$, where $\gamma_{i}>0$ is the recovery rate of the infected individuals of strain $i, i=1,2 . d_{i}>d>0$ is the death rate of the infected individuals of strain $i$, and $\alpha_{i}>0$ is the disease introduced death rate.

One of the most important concepts about the dynamics of epidemic models is the basic reproductive number $R_{0}$, which represents the expected number of secondary cases produced by a typical infected individual in a fully susceptible population $[22,23]$. According to the definition, it is easy to conclude that an epidemic will never occur when $R_{0}<1$, and instead it will occur if $R_{0}>1$. In this manuscript, we introduce two basic reproductive numbers $R_{0}^{1}$ and $R_{0}^{2}$ for our two-strain model and use these two values as the thresholds to analyze the dynamic behaviors of model (1).

This paper is organized as follows: In Section 2, we analyze the positivity and boundedness of model (1), as well as the global stability of the disease-free equilibrium. The competitive exclusion conditions are also gained in this section. In Section 3, we study the coexistence and stability of T-periodic positive solution of this model. In Section 4, the conditions of uniqueness of the solution are found. Finally, the numerical simulations are done to illustrate our results.

\section{The Global Stability of the Disease-Free Equilibrium and the Competitive Exclusion}

We first give the positivity and boundedness of model (1).

Theorem 1. Solutions of model (1) with positive initial conditions are positive and bounded.

Proof. Assume that the initial condition is $\left(S(0), I_{1}(0), I_{2}(0)\right)$ with $S(0) \geq 0, I_{1}(0) \geq 0, I_{2}(0) \geq 0$, then we have

$$
\begin{aligned}
& I_{1}(t)=\exp \left(\int_{0}^{t}\left(\frac{\beta_{1}(\tau) S(\tau)}{1+S(\tau)}-k_{1}\right) \mathrm{d} \tau\right) I_{1}(0) \geq 0, \\
& I_{2}(t)=\exp \left(\int_{0}^{t}\left(\frac{\beta_{2}(\tau) S(\tau)}{1+S(\tau)}-k_{2}\right) \mathrm{d} \tau\right) I_{2}(0) \geq 0 .
\end{aligned}
$$

The solutions $I_{1}(t)$ and $I_{2}(t)$ are nonnegative, and they are positive when $I_{1}(0) \neq 0$ and $I_{2}(0) \neq 0$.

Next, we prove the positivity of $S(t)$ with positive initial values. Assuming the contrary and letting $t_{0}>0$ be the first time such that $S\left(t_{0}\right)=0$, by the first equation of model (1), we have

$$
\left.\frac{\mathrm{d} S(t)}{\mathrm{d} t}\right|_{t=t_{0}}=\lambda+\gamma_{1} I_{1}\left(t_{0}\right)+\gamma_{2} I_{2}\left(t_{0}\right)>0,
$$

which implies $S(t)<0$ for $t \in\left(t_{0}-\varepsilon, t_{0}\right)$ and sufficient small $\varepsilon>0$. This contradicts that $t_{0}$ is the first time such that $S\left(t_{0}\right)=0$. It follows that $S(t)>0$ for $t>0$.

Next, we prove the boundedness. Let $N(t)=S(t)+$ $I_{1}(t)+I_{2}(t)$ and $\alpha$, we have

$$
\frac{\mathrm{d} N(t)}{\mathrm{d} t}=\lambda-\mathrm{d} N(t)-\alpha_{1} I_{1}(t)-\alpha_{2} I_{2}(t) \leq \lambda-\mathrm{d} N(t) .
$$

If the initial condition $0 \leq N(0) \leq(\lambda / d)$ holds, then by the comparison theorem

$$
N(t) \leq \frac{\lambda}{d}-\left(\frac{\lambda}{d}-N(0)\right) e^{-d t} \leq \frac{\lambda}{d}
$$

This inequality shows that the solution of model (1) is bounded.

This completes the proof.

Remark 1. In the rest of this paper, we assume that $\left(S(0), I_{1}(0), I_{2}(0)\right) \in D$, where $D=\left\{\left(S, I_{1}, I_{2}\right) \mid S \geq 0, I_{1} \geq 0\right.$, $\left.I_{2} \geq 0,0 \leq S+I_{1}+I_{2} \leq(\lambda / d)\right\}$.

Model (1) has a disease-free equilibrium $E_{0}=((\lambda / d), 0,0)=\left(S_{E}, 0,0\right)$. So, we define the basic reproductive number of strain $i$ as $R_{0}^{i}=\mu_{i}\left(S_{E}\right) / k_{i}$, where $\mu_{i}\left(S_{E}\right)=1 / T \int_{0}^{T}\left(\beta_{i}(t) S_{E} / 1+S_{E} d t\right),(i=1,2)$. Then, we have the following theorem.

Theorem 2. For model (1), if $R_{0}^{1}<1$ and $R_{0}^{2}<1$, then the disease-free equilibrium $E_{0}$ is globally asymptotically stable.

Proof. The solution of model (1) satisfies

$$
I_{i}(t)=I_{i}(0) \exp \left(\int_{0}^{t}\left(\frac{\beta_{i}(\tau) S(\tau)}{1+S(\tau)}-k_{i}\right) \mathrm{d} \tau\right), \quad i=1,2
$$

For any given $t \geq 0$, there exists an integer number $n$ and a real number $s$ such that $t=n T+s$, where $0 \leq s<T$ and $n \geq 0$. The inequality $S(t) \leq N(t) \leq(\lambda / d)$ implies that 


$$
\begin{aligned}
I_{i}(t) & \leq I_{i}(0) \exp \left(\int_{0}^{n T}\left(\frac{\beta_{i}(\tau) S_{E}}{1+S_{E}}-k_{i}\right) \mathrm{d} \tau\right) \exp \left(\int_{n T}^{n T+s}\left(\frac{\beta_{i}(\tau) S_{E}}{1+S_{E}}-k_{i}\right) \mathrm{d} \tau\right) \\
& =I_{i}(0) \exp \left(k_{i}\left(R_{0}^{i}-1\right) n\right) \exp \left(\frac{S_{E}}{1+S_{E}} \int_{0}^{s} \beta_{i}(\tau) d \tau-k_{i}\right),
\end{aligned}
$$

which implies that $\lim _{t \rightarrow \infty} I_{i}(t)=0$; that is, for any $\varepsilon>0$, there exists a $T_{i}>0$, such that $I_{i}(t)<\varepsilon$. Let $T_{0}=\max \left(T_{1}, T_{2}\right)$ and $\beta_{0}=\max _{0 \leq t \leq T}\left\{\beta_{1}(t)+\beta_{2}(t)\right\}$. If $t>T_{0}$, the first equation of (1) satisfies

$$
\frac{\mathrm{d} S}{\mathrm{~d} t} \geq \lambda-\beta_{0} \varepsilon-\mathrm{d} S .
$$

The comparison theorem implies that

$$
S(t) \geq \frac{\lambda-\beta_{0} \varepsilon}{d}-\left(\frac{\lambda-\beta_{0} \varepsilon}{d}-S(0)\right) e^{-\mathrm{d} t} .
$$

Since $S(t) \leq N(t) \leq \lambda / d$, we have $\lim _{t \rightarrow \infty} S(t)=(\lambda / d)$.

The limits $\lim _{t \longrightarrow \infty} S(t)=(\lambda / d)$ and $\lim _{t \longrightarrow \infty} I_{i}(t)=$ $0(i=1,2)$ show that $E_{0}$ is globally attractive.

The Jacobin matrix at the disease-free equilibrium $E_{0}=$ $\left(S_{E}, 0,0\right)$ is

$$
J=\left(\begin{array}{ccc}
-d & \gamma_{1}-\frac{\beta_{1}(t) S_{E}}{1+S_{E}} & \gamma_{2}-\frac{\beta_{2}(t) S_{E}}{1+S_{E}} \\
0 & \frac{\beta_{1}(t) S_{E}}{1+S_{E}}-k_{1} & 0 \\
0 & 0 & \frac{\beta_{2}(t) S_{E}}{1+S_{E}}-k_{2}
\end{array}\right) .
$$

The three characteristic multipliers are

$$
\begin{array}{r}
e^{-T d}, \\
e^{\int_{0}^{T}\left(\left(\beta_{1}(t) S_{E} / 1+S_{E}\right)-k_{1}\right) \mathrm{d} t}, \\
e^{\int_{0}^{T}\left(\left(\beta_{2}(t) S_{E} / 1+S_{E}\right)-k_{2}\right) \mathrm{d} t} .
\end{array}
$$

The condition $R_{0}^{i}<1(i=1,2)$ implies that if $e^{\int_{0}^{T}\left(\left(\beta_{i}(t) S_{E} / 1+S_{E}\right)-k_{i}\right) \mathrm{d} t}<1(i=1,2)$, then we claim that the disease-free equilibrium is globally asymptotically stable.

We give the competitive exclusion conditions of model (1).

Theorem 3. (i) If $\left(\beta_{1}(t) / \beta_{2}(t)\right)<\left(k_{1} / k_{2}\right)$ hold, then all solutions of model (1) with $\left(S(0), I_{1}(0), I_{2}(0)\right) \in D$ satisfies $\lim _{t \rightarrow \infty} I_{1}(t)=0$. (ii) If $\left(\beta_{1}(t) / \beta_{2}(t)\right)>\left(k_{1} / k_{2}\right)$ hold, then all solutions of model (1) with $\left(S(0), I_{1}(0), I_{2}(0)\right) \in D$ satisfies $\lim _{t \rightarrow \infty} I_{2}(t)=0$.

Proof. To prove statement (i), we let $m=\left(k_{1} / k_{2}\right)$ and consider the function

$$
V\left(S, I_{1}, I_{2}\right)=I_{1} I_{2}^{-m} .
$$

We have

$$
\frac{\mathrm{d} V\left(S, I_{1}, I_{2}\right)}{\mathrm{d} t}=\left(\beta_{1}(t)-m \beta_{2}(t)\right) \frac{S}{1+S} V\left(S, I_{1}, I_{2}\right) .
$$

The condition $\left(\beta_{1}(t) / \beta_{2}(t)\right)<m$ implies that

$$
\left(\beta_{1}(t)-m \beta_{2}(t)\right) \frac{S(t)}{1+S(t)}<0,
$$

which follows

$$
\begin{aligned}
I_{1}(t) I_{2}(t)^{-m}= & I_{1}\left(t_{0}\right) I_{2}\left(t_{0}\right)^{-m} \exp \left(\int _ { 0 } ^ { t } \left(\left(\beta_{1}(\tau)\right.\right.\right. \\
& \left.\left.\left.-m \beta_{2}(\tau)\right) \frac{S(\tau)}{1+S(\tau)}\right) \mathrm{d} \tau\right) .
\end{aligned}
$$

Let

$$
D_{0}=\left\{\left(S, I_{1}, I_{2}\right) \mid \frac{\mathrm{d} V\left(S, I_{1}, I_{2}\right)}{\mathrm{d} t}=0,\left(S, I_{1}, I_{2}\right) \in D\right\} .
$$

Then $D_{0}=\left\{\left(S, I_{1}, I_{2}\right) \mid I_{1}=0\right\}$ is an invariant set of model (1). By Lasalle's principle, we know that all solutions of model (1) in $D$ satisfy $\lim _{t \rightarrow \infty} I_{1}(t)=0$.

Using similar argument, we can obtain statement (ii).

These two conditions are usually called the principle of competitive exclusion [24, 25].

\section{Coexistence}

This section discusses the persistence of model (1).

Theorem 4. For model (1), if $R_{0}^{1}>1$ and $R_{0}^{2}>1$, then there exists a positive constant $\delta^{*}>0$, such that for at least one strain $I(t)$ satisfies $\lim \sup _{t \longrightarrow \infty} I(t) \geq \delta^{*}$.

Proof. As $S_{E}=\lambda / d$ is a global attractor for $(\mathrm{d} S / \mathrm{d} t)=\lambda-\mathrm{d} S$, we can choose small enough $\delta>0$ that for the system,

$$
\frac{\mathrm{d} S}{\mathrm{~d} t}=\lambda+\left(\gamma_{1}+\gamma_{2}-\frac{\beta_{1}(t) S}{1+S}-\frac{\beta_{2}(t) S}{1+S}\right) \eta-\mathrm{d} S .
$$

If $0<\eta<\delta$, then $\forall \varepsilon>0$. There exists a time $T$ that when $t>T,\left|S(t)-S_{E}\right|<\varepsilon$. Without loss of generality, from the conditions $R_{0}^{1}=\left(1 / k_{1} T\right) \int_{0}^{T}\left(\beta_{1}(t) S_{E} / 1+S_{E}\right)>1$ and $R_{0}^{2}=$ $\left(1 / k_{2} T\right) \int_{0}^{T}\left(\beta_{2}(t) S_{E} / 1+S_{E}\right)>1$, we can choose $\delta^{*}>0$ and small $\varepsilon$ that, for any $t>T, \quad S(t)>S_{E}-\varepsilon, \quad\left(1 / k_{1} T\right)$ $\int_{0}^{T}\left(\beta_{1}(t)\left(S_{E}-\varepsilon\right) / 1+\left(S_{E}-\varepsilon\right)\right)>1$, and $\left(1 / k_{2} T\right) \quad \int_{0}^{T} \beta_{2}(t)$ $\left(S_{E}-\varepsilon\right) / 1+\left(S_{E}-\varepsilon\right)>1$.

We prove by contradiction. Assume both of these two strains $I_{1}$ and $I_{2}$ satisfy $\lim \sup _{t \longrightarrow \infty} I_{1}(t)<\delta^{*}$ and 
$\lim \sup _{t \rightarrow \infty} I_{2}(t)<\delta^{*}$. Without loss of generality, we assume $I_{1}(t)<\delta^{*}$ and $I_{2}(t)<\delta^{*}$.

As a consequence, for all $t \geq T$, there hold

$$
\begin{aligned}
& I_{1}^{\prime}(t) \geq\left(\frac{\beta_{1}(t)\left(S_{E}-\varepsilon\right)}{1+\left(S_{E}-\varepsilon\right)}-k_{1}\right) I_{1}, \\
& I_{2}^{\prime}(t) \geq\left(\frac{\beta_{2}(t)\left(S_{E}-\varepsilon\right)}{1+\left(S_{E}-\varepsilon\right)}-k_{2}\right) I_{2},
\end{aligned}
$$

and it is easy to see that $\lim _{t \rightarrow \infty} I_{1}(t)=+\infty$ and $\lim _{t \rightarrow \infty} I_{2}(t)=+\infty$, which lead to a contradiction. Hence, the conclusion holds.

Moreover, if $\left(\beta_{2}(t) / \beta_{1}(t)\right)=\left(k_{2} / k_{1}\right)=m$ hold for all $t \geq 0$, then it is easy to get that

$$
\frac{\mathrm{d} I_{2}}{\mathrm{~d} I_{1}}=m \frac{I_{2}}{I_{1}} .
$$

Therefore,

$$
I_{2}=Q I_{1}^{m}
$$

where $Q$ is a constant which is determined by the initial values $I_{1}(0), I_{2}(0)$. Then, the dynamical behavior of model (1) is equivalent to that of the following model:

$$
\left\{\begin{array}{l}
\frac{\mathrm{d} S}{\mathrm{~d} t}=\lambda+\left(\gamma_{1}-\frac{\beta_{1}(t) S}{1+S}\right) I_{1}+Q\left(\gamma_{2}-m \frac{\beta_{1}(t) S}{1+S}\right) I_{1}^{m}-\mathrm{d} S, \\
\frac{\mathrm{d} I_{1}}{\mathrm{~d} t}=\left(\frac{\beta_{1}(t) S}{1+S}-k_{1}\right) I_{1} .
\end{array}\right.
$$

We can get the following theorem about the above model.

Theorem 5. If $R_{0}^{1}>1$, then there exists a positive constant $\delta>0$; then (1) all solutions of model (21) with positive initial conditions will satisfy $\liminf _{t \rightarrow \infty} I_{1}(t) \geq \delta$, and model (21) will admit at least one positive periodic solution.

Proof. Since $R_{0}^{1}=\mu_{1}\left(S_{E}\right) / k_{1}>1$, there exists a small enough $\eta>0$ such that $\mu_{1}\left(S_{E}-\eta\right)>k_{1}$. Let us consider the following equation:

$$
\frac{\mathrm{d} x}{\mathrm{~d} t}=\lambda-\beta_{1}(t) \delta-m Q \beta_{1}(t) \delta^{m}-\mathrm{d} x,
$$

and the solution of this equation (22) is

$$
x(t)=S_{E}+C_{0} e^{-d t}-\left(\delta+m Q \delta^{m}\right) \int_{0}^{t} \beta_{1}(\tau) e^{d(\tau-t)} \mathrm{d} \tau,
$$

where $C_{0}$ is a constant. As $0<\beta_{1}(t)<M$, the following inequalities hold:

$$
S_{E}-\left(\delta+m Q \delta^{m}\right) \frac{M}{d} \leq \lim _{t \longrightarrow \infty} x(t) \leq S_{E}
$$

Thus, we can fix a small enough number $\delta=\delta^{*}>0$ such that $\lim _{t \rightarrow \infty} x(t)>S_{E}-\eta$.
Suppose, by contradiction, that $\limsup _{t \rightarrow \infty} I_{1}(t)<\delta^{*}$. Without loss of generality, we can assume that $I_{1}(t)<\delta^{*}$, it follows $0 \leq I_{1}(t)<\delta^{*}$. By the first equation of model (21), we have the following inequality:

$$
S^{\prime}(t) \geq \lambda-\beta_{1}(t) \delta^{*}-m Q \beta_{1}(t) \delta^{* m}-\mathrm{d} S .
$$

Thus, there exists a time $T^{*}$; for all $t>T^{*}$, we have $S(t)>S_{E}-\eta$. As a consequence, for all $t \geq T$, there holds

$$
I_{1}^{\prime}(t) \geq\left(\frac{\beta(t)\left(S_{E}-\eta\right)}{1+\left(S_{E}-\eta\right)}-k\right) I_{1} .
$$

Since $\mu\left(S_{E}-\eta\right)>k$, it is easy to see that $\lim _{t \rightarrow \infty} I_{1}(t)=$ $+\infty$, which leads to a contradiction. Hence, $\lim \sup _{t \rightarrow \infty} I_{1}$ $(t) \geq \delta^{*}$.

Define

$$
\begin{aligned}
X & =R_{+}^{2}, \\
X_{0} & =R_{+} \times \operatorname{int} R_{+}, \\
\partial X_{0} & =X / X_{0},
\end{aligned}
$$

where $R_{+}=\{x \mid x \geq 0\}$ and int $R_{+}=\{x \mid x>0\}$. Then, assume $u\left(t,\left(S^{0}, I_{1}^{0}\right)\right)$ is the solution of model $(21)$ and $p\left(\left(S^{0}, I_{1}^{0}\right)\right)=$ $u\left(T,\left(S^{0}, I_{1}^{0}\right)\right)$ is the Poincare map.

$E_{0}$ is globally attractive in $\partial X_{0}$ for $p$, and $E_{0}$ is isolated invariant sets in $X$ with $W^{s}\left(E_{0}\right) \cap X_{0}=\varnothing$. Clearly, every orbit in $\partial X_{0}$ converges to $E_{0}$. By Theorem 1.3.1 in [26], for a stronger repelling property of $\partial X_{0}$, we conclude that $p$ is uniformly persistent with respect to $\left(X_{0}, \partial X_{0}\right)$. Thus, Theorem 3.1.1 in [26] implies the uniform persistence of the solutions of model (21) with respect to $\left(X_{0}, \partial X_{0}\right)$. By Theorem 1.3.6 in [26], $p$ has a fixed point $\left(\widehat{S}(0), \widehat{I}_{1}(0)\right) \in X_{0}$. Then $\widehat{S}(0) \in R^{+}$and $\widehat{I}_{1}(0) \in \operatorname{int} R^{+}$. We further claim that $\widehat{S}(0) \in R_{+} /\{0\}$. Suppose $\widehat{S}(0)=0$, then we have $\lambda+\gamma_{1} \widehat{I_{1}}(0)+\gamma_{2} Q \widehat{I}_{1}^{m}(0)=0$, which is a contradiction. So from model $(21)$, we can get $u(t,(\widehat{S}(0)$, $\left.\left.\widehat{I_{1}}(0)\right)\right) \in \operatorname{int}\left(R_{+}^{2}\right), \forall t>0$. Then $\left(\widehat{S}(0), \widehat{I_{1}}(0)\right)$ is a componentwise positive fixed point of $p$. Thus, $\left(\widehat{S}(t), \widehat{I}_{1}(t)\right)$ is a positive T-periodic solution of model (21).

Remark 2. It is easy to conclude that when the condition $\forall t,\left(\beta_{2}(t) / \beta_{1}(t)\right)=\left(k_{2} / k_{1}\right)=m$ and $R_{0}^{1}>1$ hold, there exist at least one persistent solution $\left(S(t), I_{1}(t)\right.$, and $\left.I_{2}(t)\right)$ with respect to $I_{2}(t)=Q I_{1}(t)^{m}$ and $Q \geq 0$. Moreover, when $Q=0$, model (21) becomes the plane system that $I_{2}(t)=0$.

Then, we consider a more special case that the infection rate of model (21) satisfies $\beta_{1}(t)=b_{0}+\varepsilon \cos (t)$, where $\varepsilon>0$ is a small number. When $R_{0}^{1}>1$ and $\varepsilon=0$, model (21) becomes an autonomous model

$$
\left\{\begin{array}{l}
\frac{\mathrm{d} S}{\mathrm{~d} t}=\lambda+\left(\gamma_{1}-\frac{b_{0} S}{1+S}\right) I_{1}+Q\left(\gamma_{2}-m \frac{b_{0} S}{1+S}\right) I_{1}^{m}-\mathrm{d} S, \\
\frac{\mathrm{d} I_{1}}{\mathrm{~d} t}=\left(\frac{b_{0} S}{1+S}-k_{1}\right) I_{1} .
\end{array}\right.
$$

The positive equilibrium $E_{1}=\left(S^{*}, I^{*}\right)$ for model (28) satisfies 


$$
\begin{gathered}
\frac{b_{0} S^{*}}{1+S^{*}}=k_{1}, \\
\left(\gamma-\frac{b_{0} S^{*}}{1+S^{*}}\right) I^{*}+Q\left(\gamma_{2}-m \frac{b_{0} S^{*}}{1+S^{*}}\right) I^{* m}=\mathrm{d} S^{*}-\lambda .
\end{gathered}
$$

As $\left(b_{0} S^{*} / 1+S^{*}\right)=k_{1}>\gamma_{1}, \quad m\left(b_{0} S^{*} / 1+S^{*}\right)=k_{2}>\gamma_{2}$ and $R_{0}^{1}>1$ implies $\mathrm{d} S^{*}-\lambda>0$, so there exists unique positive equilibrium $E_{1}=\left(S^{*}, I^{*}\right)$ for model (28). It is easy to check the Jacobin matrix at equilibrium $E_{1}$ is

$$
\left(\begin{array}{cc}
-\frac{b_{0} I^{*}}{\left(1+S^{*}\right)^{2}}-\frac{m Q b_{0} I^{* m}}{\left(1+S^{*}\right)^{2}}-d & \gamma_{1}-\frac{b_{0} S^{*}}{1+S^{*}}+m Q\left(\gamma_{2}-m \frac{b_{0} S^{*}}{1+S^{*}}\right) I_{1}^{*}(m-1) \\
\frac{b_{0} I^{*}}{\left(1+S^{*}\right)^{2}} & 0
\end{array}\right)
$$

and its two eigenvalues $\lambda_{1}, \lambda_{2}$ satisfy

$$
\begin{aligned}
\lambda_{1}+\lambda_{2}= & -\frac{b_{0} I^{*}}{\left(1+S^{*}\right)^{2}}-\frac{m Q b_{0} I^{* m}}{\left(1+S^{*}\right)^{2}}-d<0, \\
\lambda_{1} \lambda_{2}= & \frac{b_{0} I^{*}}{\left(1+S^{*}\right)^{2}}\left(\gamma_{1}-\frac{b_{0} S^{*}}{1+S^{*}}\right. \\
& \left.+m Q\left(\gamma_{2}-m \frac{b_{0} S^{*}}{1+S^{*}}\right) I_{1}^{*(m-1)}\right)>0,
\end{aligned}
$$

when $R_{0}^{1}>1$, which implies that the real parts of $\lambda_{1}$ and $\lambda_{2}$ are negative. So the unique equilibrium $E_{1}$ is asymptotically stable.

The right-hand side of model (21) $f(S, I, \mu)$ can be illustrated as

$$
\begin{aligned}
f\left(S, I, b_{1}\right)= & \left(\begin{array}{c}
\lambda+\left(\gamma_{1}-\frac{b_{0} S}{1+S}\right) I_{1}+Q\left(\gamma_{2}-m \frac{b_{0} S}{1+S}\right) I_{1}^{m}-d S \\
\left(\frac{b_{0} S}{1+S}-k_{1}\right) I_{1}
\end{array}\right) \\
& +\varepsilon\left(\begin{array}{c}
-\frac{\cos (t) S I_{1}}{1+S}-Q m \frac{\cos (t) S I_{1}^{m}}{1+S} \\
\frac{\cos (t) S I_{1}}{1+S}
\end{array}\right) .
\end{aligned}
$$

It is easy to see $f(S, I, \varepsilon)$ is analytic with $S, I \in R^{+}$and $\varepsilon \in R$, and when $\epsilon=0$, model (21) becomes (28). Then by Theorem 1.1 in Chapter 14 in [27], there exists a $T$-periodic solution $(S(t), I(t))$ near equilibrium $E_{1}$ for small $\varepsilon$, and by Theorem 1.2 in Chapter 14 in [27], this solution $(S(t), I(t))$ is asymptotically stable.

Thus, we have the following theorem.

Theorem 6. For model (21), if $R_{0}^{1}>1$ and $\beta_{1}(t)=b_{0}+$ $\varepsilon \cos (t)$, where $\varepsilon>0$ and small, then there exists a asymptotically stable positive $2 \pi$-periodic solution $(S(t), I(t))$ for model (21).

\section{Uniqueness of the Solution on the Surface}

In this section, we mainly talk about the uniqueness of the solution of model (21), and the solution is on the surface $I_{2}=Q I_{1}^{m}$. We consider the model

$$
\left\{\begin{array}{l}
\frac{\mathrm{d} S}{\mathrm{~d} t}=\lambda+\left(\gamma_{1}-\frac{\beta(t) S}{1+S}\right) I+Q\left(\gamma_{2}-\frac{m \beta(t) S}{1+S}\right) I^{m}-\mathrm{d} S \\
\frac{\mathrm{d} I}{\mathrm{~d} t}=\left(\frac{\beta(t) S}{1+S}-k\right) I,
\end{array}\right.
$$

where $\beta(t)=b_{0}+\varepsilon \cos (t)$ and $b_{0}=8, \lambda=1, \gamma_{1}=\gamma_{2}=1$, $d=1, k=2, Q=2$, and $m=2$.

Theorem 7. When $\varepsilon$ is small enough, model (5) exhibits unique $2 \pi$-periodic solution.

Proof. Assume system (33) has a solution

$$
\begin{aligned}
& S_{p}(t)=S_{0}(t)+\varepsilon S_{1}(t)+\varepsilon^{2} S_{2}(t)+\varepsilon^{3} S_{3}(t)+\ldots, \\
& I_{p}(t)=I_{0}(t)+\varepsilon I_{1}(t)+\varepsilon^{2} I_{2}(t)+\varepsilon^{3} I_{3}(t)+\ldots
\end{aligned}
$$

Using (34) in model (35), and equating the powers of $\varepsilon$, we get the following equations:

$$
\begin{aligned}
& \left\{\begin{array}{l}
\frac{\mathrm{d} S_{0}(t)}{\mathrm{d} t}=\lambda+\left(\gamma_{1}-\frac{b_{0} S_{0}(t)}{1+S_{0}(t)}\right) I_{0}(t) \\
+Q\left(\gamma_{2}-\frac{m b_{0} S_{0}(t)}{1+S_{0}(t)}\right) I_{0}(t)^{m}-\mathrm{d} S_{0}(t), \\
\frac{\mathrm{d} I_{0}(t)}{\mathrm{d} t}=\left(\frac{b_{0} S_{0}(t)}{1+S_{0}(t)}-k\right) I_{0}(t), \\
\left.\frac{\mathrm{d} S_{j}(t)}{\mathrm{d} t}\right)=J\left(\begin{array}{c}
S_{j}(t) \\
I_{j}(t)
\end{array}\right)+\left(\begin{array}{c}
F_{j}(t) \\
G_{j}(t)
\end{array}\right),
\end{array}\right.
\end{aligned}
$$


where $F_{j}$ and $G_{j}$ are periodic functions of the period $2 \pi$, and they are only relevant to $S_{0}(t), I_{0}(t), \ldots, S_{j-1}(t), I_{j-1}(t)$, and

$$
J=\left(\begin{array}{cc}
-2 \frac{b_{0} Q I_{0}(t)^{2}}{\left(1+S_{0}(t)\right)^{2}}-\frac{b_{0} I_{0}(t)}{\left(1+S_{0}(t)\right)^{2}}-d & \gamma_{1}-\frac{b_{0} S_{0}(t)}{1+S_{0}(t)}+2 Q\left(\gamma_{2}-2 \frac{b_{0} S_{0}(t)}{1+S_{0}(t)}\right) I_{0}(t) \\
\frac{b_{0} I_{0}(t)}{\left(1+S_{0}(t)\right)^{2}} & \frac{b_{0} S_{0}(t)}{1+S_{0}(t)}-k
\end{array}\right)
$$

We set $b_{0}=8, \lambda=1, \gamma_{1}=\gamma_{2}=1, d=1, k=2$, and $Q=$ 2 and obtain

$$
\begin{aligned}
& S_{0}(t)=\frac{1}{3}, \\
& I_{0}(t)=\frac{1}{3},
\end{aligned}
$$

The fundamental matrix $\Phi(t)$ of the linear homogeneous differential equation system of system (35) is

$$
\Phi(t)=\left(\begin{array}{l}
\phi_{11}(t), \phi_{12}(t) \\
\phi_{21}(t), \phi_{22}(t)
\end{array}\right)
$$

where

$$
\begin{aligned}
& \phi_{11}(t)=e^{-7 / 4 t} \cos (1 / 4 t \sqrt{23})-\frac{7 \sqrt{23} e^{-7 / 4 t} \sin (1 / 4 t \sqrt{23})}{23}, \\
& \phi_{12}(t)=-\frac{12 \sqrt{23} e^{-7 / 4 t} \sin (1 / 4 t \sqrt{23})}{23}, \\
& \phi_{21}(t)=\frac{6 \sqrt{23} e^{-7 / 4 t} \sin (1 / 4 t \sqrt{23})}{23}, \\
& \phi_{22}(t)=e^{-7 / 4 t} \cos (1 / 4 t \sqrt{23})+\frac{7 \sqrt{23} e^{-7 / 4 t} \sin (1 / 4 t \sqrt{23})}{23} .
\end{aligned}
$$

The inverse matrix of $\Phi(t)$ is $\Psi(t)$ :

$$
\Psi(t)=\left(\begin{array}{l}
\psi_{11}(t), \psi_{12}(t) \\
\psi_{21}(t), \psi_{22}(t)
\end{array}\right)
$$

where

$$
\begin{aligned}
& \psi_{11}(t)=\frac{1}{23} \frac{7 \sqrt{23} \sin (1 / 4 t \sqrt{23})+23 \cos (1 / 4 t \sqrt{23})}{e^{-7 / 4 t}\left((\cos (1 / 4 t \sqrt{23}))^{2}+(\sin (1 / 4 t \sqrt{23}))^{2}\right)}, \\
& \psi_{12}(t)=\frac{12 \sqrt{23} \sin (1 / 4 t \sqrt{23})}{23 e^{-7 / 4 t}\left((\cos (1 / 4 t \sqrt{23}))^{2}+(\sin (1 / 4 t \sqrt{23}))^{2}\right)}, \\
& \psi_{21}(t)=-\frac{6 \sqrt{23} \sin (1 / 4 t \sqrt{23})}{23 e^{-7 / 4 t}\left((\cos (1 / 4 t \sqrt{23}))^{2}+(\sin (1 / 4 t \sqrt{23}))^{2}\right)}, \\
& \psi_{22}(t)=\frac{1}{23} \frac{-7 \sqrt{23} \sin (1 / 4 t \sqrt{23})+23 \cos (1 / 4 t \sqrt{23})}{e^{-7 / 4 t}\left((\cos (1 / 4 t \sqrt{23}))^{2}+(\sin (1 / 4 t \sqrt{23}))^{2}\right)}
\end{aligned}
$$

We obtain the solution of system (35)

$$
\left(\begin{array}{c}
S_{j}(t) \\
I_{j}(t)
\end{array}\right)=\Phi(t)\left(\begin{array}{c}
c_{1 j} \\
c_{2 j}
\end{array}\right) \Psi(t) \int_{0}^{t} \Psi(\tau)\left(\begin{array}{c}
F_{j}(\tau) \\
G_{j}(\tau)
\end{array}\right) \mathrm{d} \tau .
$$

By periodicity, we obtain

$$
\left(\begin{array}{c}
c_{1 j} \\
c_{2 j}
\end{array}\right)=(\Phi(0)-\Phi(2 \pi))^{-1} \Phi(2 \pi) \int_{0}^{2 \pi} \Psi(\tau)\left(\begin{array}{c}
F_{j}(\tau) \\
G_{j}(\tau)
\end{array}\right) \mathrm{d} \tau .
$$

After simple calculation, we get $\operatorname{det}(\Phi(0)-\Phi(2 \pi))^{-1} \approx$ $1 \neq 0$. Therefore, we obtain the unique existence of the periodic solution of period $2 \pi$ of model (33).

\section{Numerical Simulation}

Numerical simulations are performed to illustrate the dynamic behaviors of model (1) and (21).

Figures 1(a) and 1(b) show the solution of (21) under the conditions $R_{0}^{1}=1.5$ and $R_{0}^{1}=2$. From these two figures, we can see that there exists one stable periodic solution of model (21) under different values of $R_{0}$, and when the value of $R_{0}$ becomes larger, the mean value of $I(t)$ becomes larger.

Figures 2(a) and 2(b) show the results of Theorem 3. When the competitive exclusion condition holds, one strain will drive another into extinction.

Figures 3(a) and 3(b) show the coexistence of model (1) under the condition $\beta_{1}(t) / k_{1}=\beta_{2}(t) / k_{2}$. Figure $3(\mathrm{a})$ is under the initial condition $\left(S(0), I_{1}(0), I_{2}(0)\right)=(2,1,2)$ and Figure $3(\mathrm{~b})$ is $\left(\left(S(0), I_{1}(0), I_{2}(0)\right)=(2,2,1)\right.$.

Figure 4(a) is the solution of model (1) with $\beta_{1}=3+$ $\sin (t), \beta_{2}=3+\cos (t), \lambda=1, d=1, k_{1}=1, \gamma_{1}=0.1, k_{2}=$ $1, \gamma_{2}=0.1$, and $R_{0}^{1}=R_{0}^{2}$. Figure 4(b) is the solution of model (1) with $\beta_{1}=3+\sin (t), \beta_{2}=3.5+\sin (t), \lambda=1, \quad d=1$, $k_{1}=1.2, \gamma_{1}=0.1, k_{2}=1.401, \gamma_{2}=0.1, \quad R_{0}^{1}=1.25, \quad R_{0}^{2}=$ 1.2491 , and $R_{0}^{1} \neq R_{0}^{2}$. These two figures illustrate that the basic reproductive numbers of different strains cannot determine the coexistence of model (1). One strain can drive another into extinction even with the same reproductive number, and the two strains can coexist with different basic reproductive numbers.

\section{Discussion}

In this paper, we study the dynamical behaviors of a twostrain SIS model with a periodic infection rate and get its 


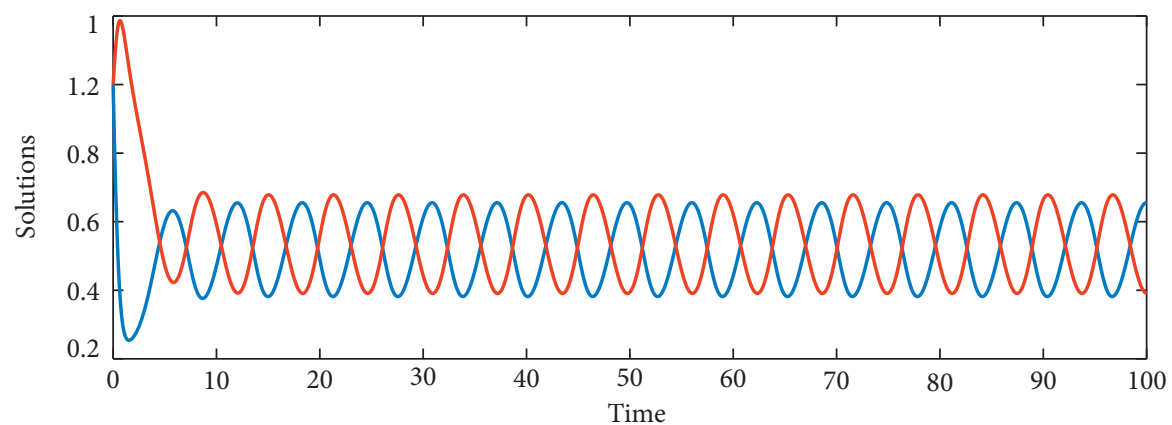

$-S$

(a)

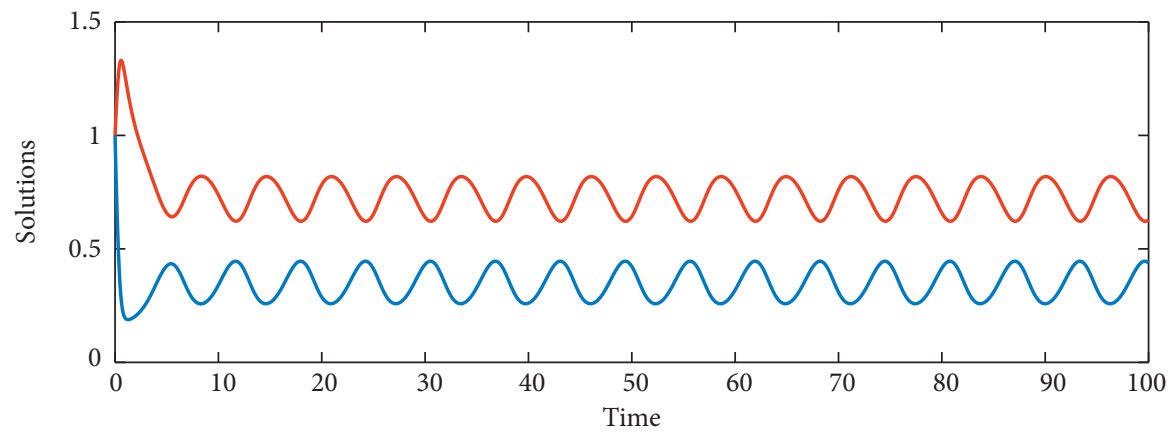

- $^{s}$

(b)

Figure 1: The simulated solution of model (21) under the conditions (a) $R_{0}^{1}=1.5$ and (b) $R_{0}^{1}=2$.

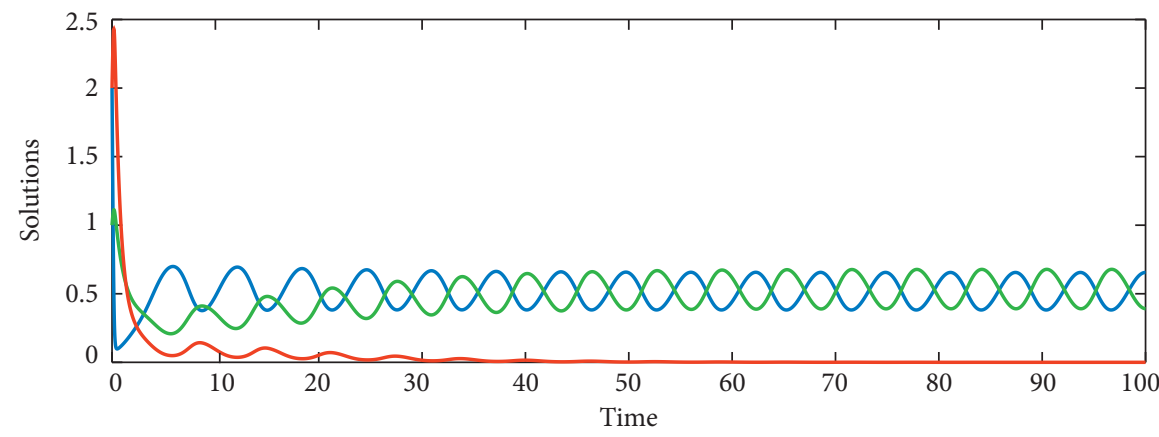

$-S$
$-\quad I_{1}$
$-\quad I_{2}$

(a)

FIgUre 2: Continued. 


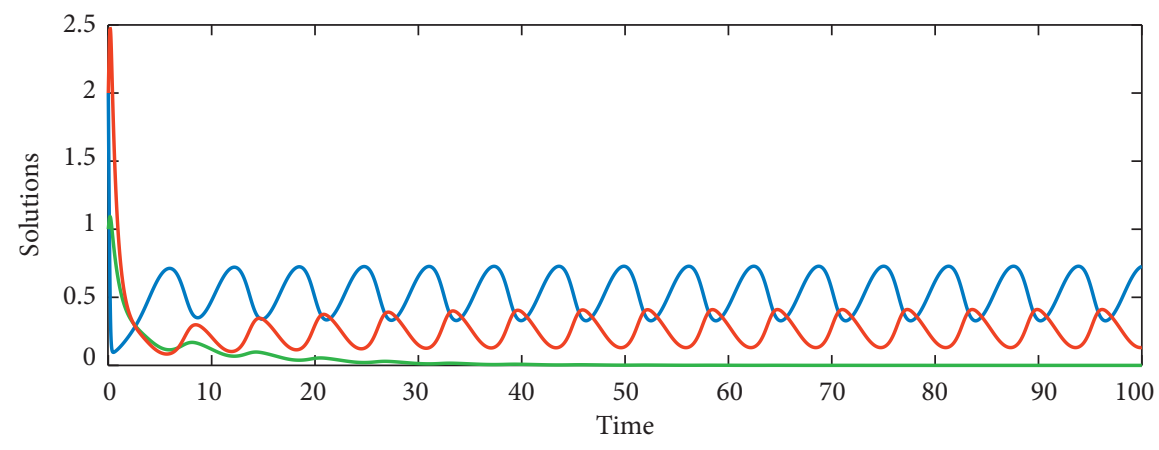

$$
\begin{array}{rr}
- & S \\
- & I_{1} \\
- & I_{2}
\end{array}
$$

(b)

Figure 2: The simulated solution of model (1) under the conditions (a) $\beta_{1}(t) / k_{1}=3+\sin (t)$ and $\beta_{2}(t) / k_{2}=2.85+0.95 \sin (t)$ and (b) $\beta_{1}(t) / k_{1}=2.72+0.91 \sin (t)$ and $\beta_{2}(t) / k_{2}=3+\sin (t)$.
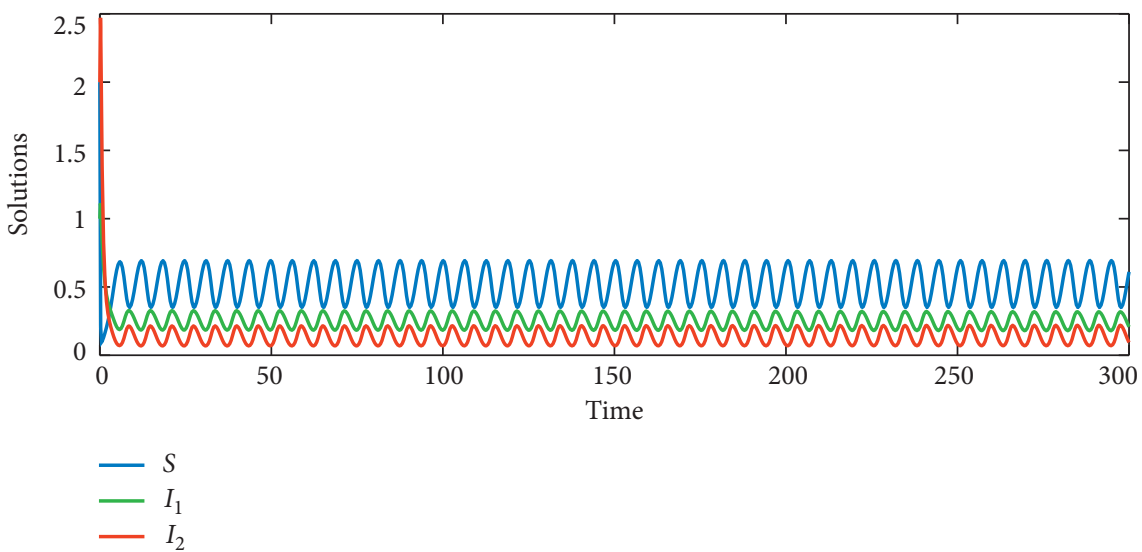

(a)

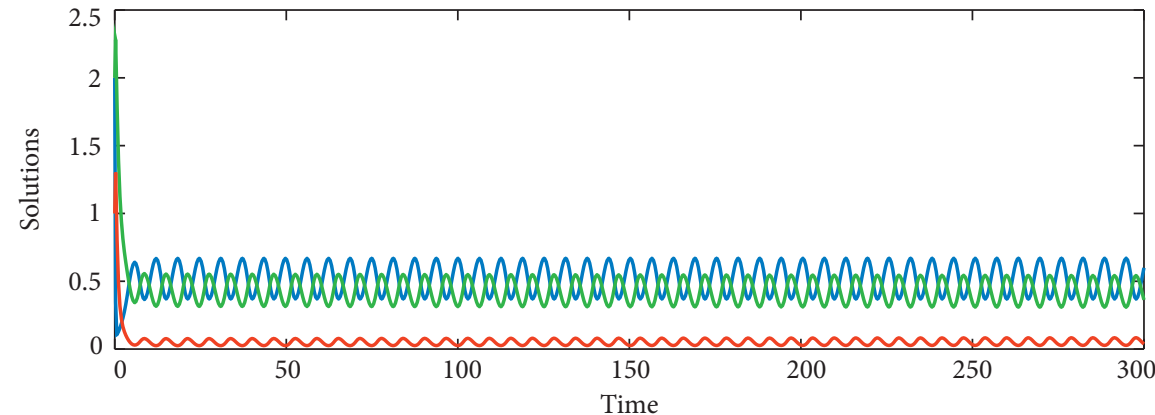

$-S$
$-\quad I_{1}$
$-I_{2}$

(b)

Figure 3: The simulated solution of model (1) with $\beta_{1}(t) / k_{1}=\beta_{2}(t) / k_{2}=3+\sin (t)$ and the initial conditions (a) $\left(S(0), I_{1}(0), I_{2}(0)\right)=$ $(2,1,2)$ and $(b)\left(\left(S(0), I_{1}(0), I_{2}(0)\right)=(2,2,1)\right.$.

persistence, competitive exclusion, and coexistence conditions. We gain the global stability conditions of the diseasefree equilibrium $E_{0}$ and establish the competitive exclusion condition of the two strains. The coexistence and uniqueness are also discussed and proved. Our results lead to a new insight into the mechanism of two strains interaction and provide a new approach to investigate the inference of the periodic infection rate on the coexistence of two strains. It is 

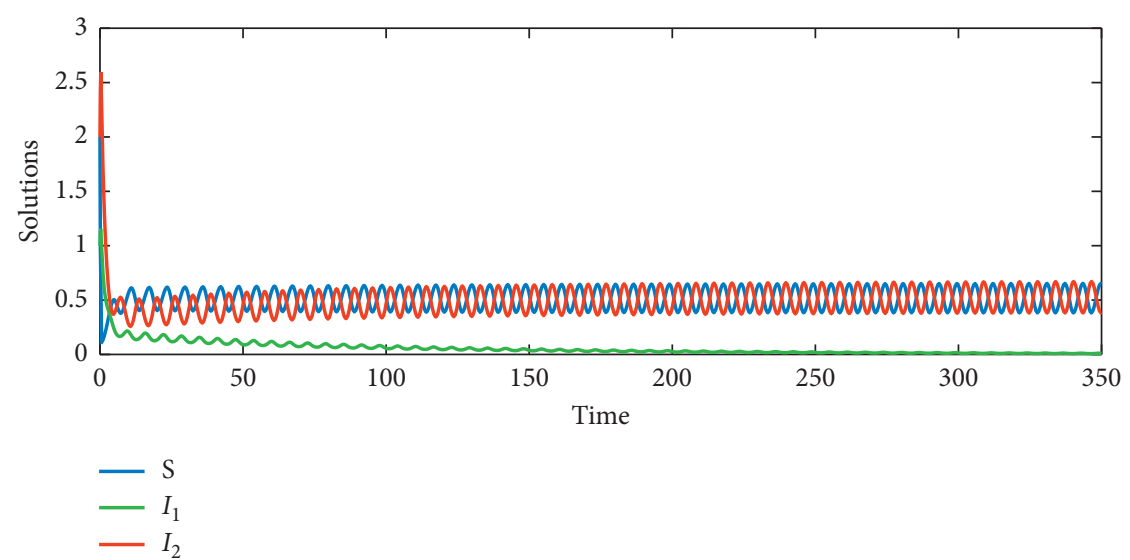

(a)

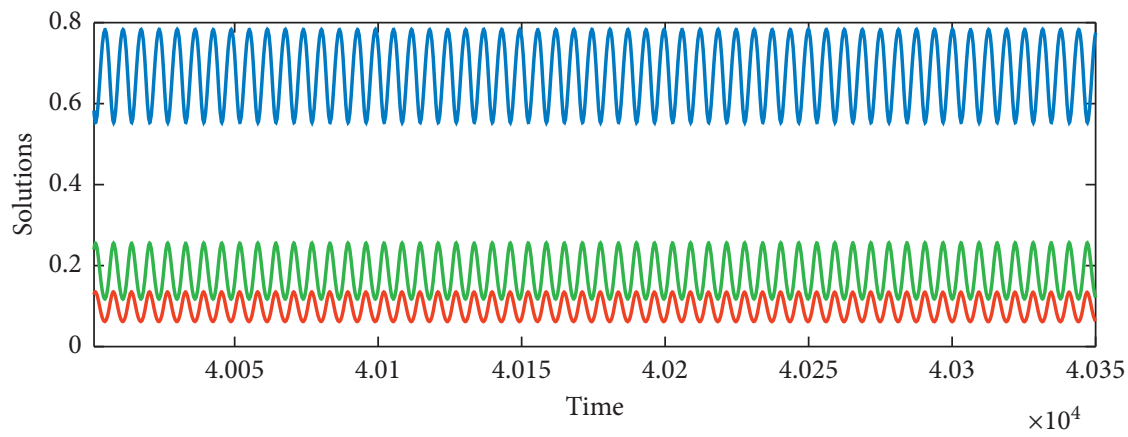

(b)

Figure 4: (a) The simulated solution of model (1) with $\beta_{1}=3+\sin (t), \beta_{2}=3+\cos (t), \lambda=1, d=1, k_{1}=1, \gamma_{1}=0.1, k_{2}=1$, and $\gamma_{2}=0.1$. (b) The stable simulated solution of model (1) with $\beta_{1}=3+\sin (t), \beta_{2}=3.5+\sin (t), \lambda=1, d=1, k_{1}=1.2, \gamma_{1}=0.1, k_{2}=1.401$, and $\gamma_{2}=0.1$.

worth to mention that the analysis in this article can be applied to the $n$-strain epidemic model with a periodic infection rate and obtain similar results.

\section{Data Availability}

No data were used to support this study.

\section{Conflicts of Interest}

The authors declare that they have no conflicts of interest.

\section{References}

[1] W. O. Kermack and A. G. McKendrick, "A contribution to the mathematical theory of epidemics," Proceedings of the Royal Society of London. Series A, Containing Papers of a Mathematical and Physical Character, vol. 115, no. 772, pp. 700-721, 1927.

[2] X. Du, A. A. King, R. J. Woods, and M. Pascual, "Evolutioninformed forecasting of seasonal influenza A (H3N2)," Science Translational Medicine, vol. 9, no. 413, Article ID eaan5325, 2017.
[3] X. Du and M. Pascual, "Incidence prediction for the 20172018 influenza season in the United States with an evolutioninformed model," PLoS Currents, vol. 10, 2018.

[4] S. Tang, Y. Xiao, and D. Clancy, "New modelling approach concerning integrated disease control and cost-effectivity," Nonlinear Analysis: Theory, Methods \& Applications, vol. 63, no. 3, pp. 439-471, 2005

[5] Y. Xiao, S. Tang, and J. Wu, "Media impact switching surface during an infectious disease outbreak," Scientific Reports, vol. 5, p. 7838, 2015.

[6] Y. Xiao, X. Xu, and S. Tang, "Sliding mode control of outbreaks of emerging infectious diseases," Bulletin of Mathematical Biology, vol. 74, no. 10, pp. 2403-2422, 2012.

[7] Y. A. Kuznetsov and C. Piccardi, "Bifurcation analysis of periodic SEIR and SIR epidemic models," Journal of Mathematical Biology, vol. 32, no. 2, pp. 109-121, 1994.

[8] W. Wang and X.-Q. Zhao, "Threshold dynamics for compartmental epidemic models in periodic environments," Journal of Dynamics and Differential Equations, vol. 20, no. 3, pp. 699-717, 2008.

[9] F. Zhang and X.-Q. Zhao, "A periodic epidemic model in a patchy environment," Journal of Mathematical Analysis and Applications, vol. 325, no. 1, pp. 496-516, 2007. 
[10] USA CDC, "Situation update: summary of weekly fluview report," USA CDC, Atlanta, GA, USA, 2019, https://www.cdc. gov/flu/weekly/summary.htm.

[11] P. E. M. Fine and J. A. Clarkson, "Measles in England and Wales-I: an analysis of factors underlying seasonal patterns," International Journal of Epidemiology, vol. 11, no. 1, pp. 5-14, 1982.

[12] A. C. Lowen, S. Mubareka, J. Steel, and P. Palese, "Influenza virus transmission is dependent on relative humidity and temperature," PLoS Pathogens, vol. 3, no. 10, p. e151, 2007.

[13] J. Shaman and M. Kohn, "Absolute humidity modulates influenza survival, transmission, and seasonality," Proceedings of the National Academy of Sciences, vol. 106, no. 9, pp. 3243-3248, 2009.

[14] K. Dietz, "The incidence of infectious diseases under the influence of seasonal fluctuations," in Mathematical Models in Medicine, pp. 1-15, Springer, Berlin, Germany, 1976.

[15] H. W. Hethcote, "Asymptotic behavior in a deterministic epidemic model," Bulletin of Mathematical Biology, vol. 35, no. 5-6, pp. 607-614, 1973.

[16] H. J. Bremermann and H. R. Thieme, "A competitive exclusion principle for pathogen virulence," Journal of Mathematical Biology, vol. 27, no. 2, pp. 179-190, 1989.

[17] R. M. May and A. N. Martin, "Coinfection and the evolution of parasite virulence," Proceedings of the Royal Society of London. Series B: Biological Sciences, vol. 261, no. 1361, pp. 209-215, 1995.

[18] J. Li, Y. Zhou, Z. Ma, and H. J. M. Hyman, "Epidemiological models for mutating pathogens," SIAM Journal on Applied Mathematics, vol. 65, no. 1, pp. 1-23, 2004.

[19] M. Nuño, Z. Feng, M. Martcheva, and C. Castillo-Chavez, "Dynamics of two-strain influenza with isolation and partial cross-immunity," SIAM Journal on Applied Mathematics, vol. 65, no. 3, pp. 964-982, 2005.

[20] M. Martcheva, "A non-autonomous multi-strain SIS epidemic model," Journal of Biological Dynamics, vol. 3, no. 2-3, pp. 235-251, 2009.

[21] C. Castillo-Chavez, W. Huang, and J. Li, "Competitive exclusion and coexistence of multiple strains in an SIS STD model," Siam Journal on Applied Mathematics, vol. 59, no. 5, pp. 1790-1811, 1999.

[22] O. Diekmann, J. A. P. Heesterbeek, and J. A. J. Metz, "On the definition and the computation of the basic reproduction ratio $R_{0}$ in models for infectious diseases in heterogeneous populations," Journal of Mathematical Biology, vol. 28, no. 4, pp. 365-382, 1990.

[23] T. Kuniya, "Global behavior of a multi-group sir epidemic model with age structure and an application to the chlamydia epidemic in Japan," SIAM Journal on Applied Mathematics, vol. 79, no. 1, pp. 321-340, 2019.

[24] M. Martcheva, An Introduction to Mathematical Epidemiology, Springer, Berlin, Germany, 2015.

[25] Y. Wang and $\mathrm{H}$. Wu, "Global dynamics of parasitism-competition systems with one host and multiple parasites," Journal of Theoretical Biology, vol. 461, pp. 268-275, 2019.

[26] X.-Q. Zhao, J. Borwein, and B. Peter, Dynamical Systems in Population Biology, Springer, Berlin, Germany, 2003.

[27] E. A. Coddington and N. Levinson, Theory of Ordinary Differential Equations, Tata McGraw-Hill Education, New York, NY, USA, 1955. 